\title{
Pneumonia grave por "Chlamydia psittaci" "
}

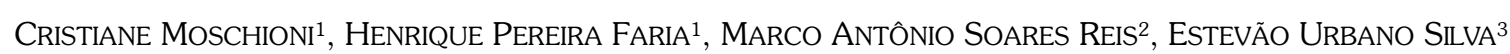

A psitacose, também conhecida como ornitose, é causada pela Chlamydia psittaci; caracteriza-se por doença de início insidioso, sintomas brandos e inespecíficos, lembrando infecção de vias aéreas superiores. Acomete principalmente o pulmão, sendo raramente doença sistêmica e fatal. Descreve-se um caso raro de pneumonia por Chlamydia psittaci que evoluiu para insuficiência respiratória aguda, necessitando de ventilação mecânica. Destaca-se a importância em considerar o diagnóstico, especialmente em casos de pneumonia comunitária que evolui de modo insatisfatório, que não responde à terapia antimicrobiana e cuja epidemiologia é positiva para exposição às aves. $\mathrm{O}$ diagnóstico precoce é fundamental devido à excelente resposta terapêutica. O diagnóstico tardio pode levar a curso grave e fatal da doença. (J Pneumol 2001;27(4):219-222)

\section{Severe pneumonia due to Chlamydia psittaci}

Psittacosis, also known as ornithosis, is a disease caused by Chlamydia psittaci. The most common clinical presentation is insidious onset, mild symptoms resembling a nonspecific viral illness and preference for the lungs. It is rarely a systemic and fatal disease. It is described a rare case of pneumonia due to Chlamydia psittaci that progressively developed into respiratory failure, requiring mechanical ventilation. It is very important to consider psittacosis in cases of atypical pneumonia whose evolution is unsatisfactory, with no response to antimicrobial therapy and epidemiology is positive for exposure to birds. Prompt recognition is vital as the response to appropriate treatment is excellent. Delayed diagnosis may lead to a severe course and fatal outcome.

Descritores - Pneumonia por micoplasma. Ornitose.

Key words - Mycoplasma pneumonia. Ornithosis.

\section{INTRODUÇÃO}

Em 1893, Morange descreveu um agente infeccioso transmitido por papagaios que causava, em humanos, infecção no trato respiratório semelhante à gripe $e^{(1)}$. Denominou essa doença de psitacose, da palavra grega psitta-

* Trabalho realizado no Centro de Tratamento Intensivo do Hospital Universitário São José (HUSJ), Belo Horizonte, MG.

1. Médico Residente do Serviço de Clínica Médica.

2. Especialista em Clínica Médica, Pneumologia e Medicina Intensiva; Coordenador do Centro de Tratamento Intensivo.

3. Infectologista; Coordenador do Serviço de Controle de Infecções Hospitalares.

Endereço para correspondência - Rua Gonçalves Dias, 750/1.501 30140-091 - Belo Horizonte, MG. Tel. (31) 3261-2300; E-mail: cristianemoschioni@bol.com.br

Recebido para publicação em 28/9/00. Aprovado, após revisão, em 1/3/01.
Siglas e abreviaturas utilizadas neste trabalho

FC - Freqüência cardíaca

Bpm - Batimentos por minuto

FR - Freqüência respiratória

Ipm - Incursões por minuto

$\mathrm{PaO}_{2}$ - Pressão parcial de oxigênio no sangue arterial

$\mathrm{PaCO}_{2}$ - Pressão parcial de gás carbônico no sangue arterial

$\mathrm{SatO}_{2}-$ Saturação de oxigênio

$\mathrm{HCO}_{3}$ - Bicarbonato

$\mathrm{BE}-$ Excesso de base

cus, papagaio. Em 1930, o microrganismo foi identificado em vários laboratórios e posteriormente incluído no gênero Chlamydia e na espécie Chlamydia psittaci. Atualmente, mais de 130 espécies de aves já foram documentadas como hospedeiras da bactéria ${ }^{(2)}$. A maioria das infecções em humanos resulta do contato com pássaros infectados e decorre da inalação do microrganismo ${ }^{(3,4)}$. O nome psitacose persistiu, apesar de o termo ornitose descrever com mais exatidão o potencial que qualquer pássaro tem de ser um vetor na transmissão da doença ${ }^{(5)}$. 


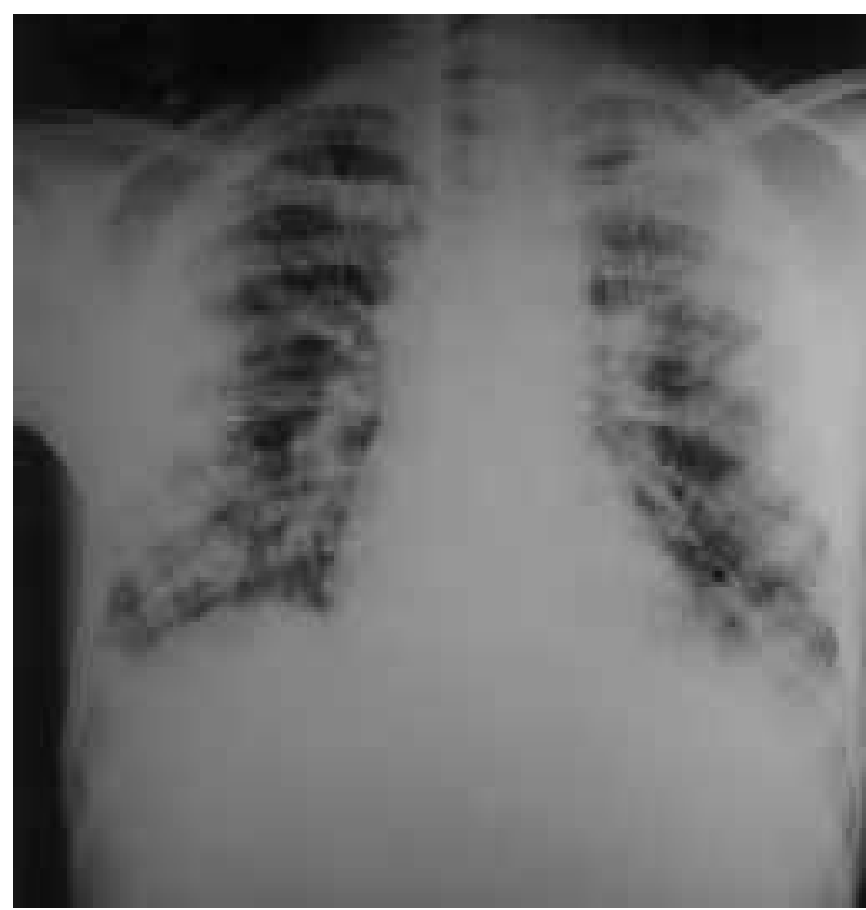

Figura 1 - Pneumonia por Chlamydia psittaci. Radiografia de tórax à admissão do paciente. Nota-se infiltrado pulmonar difuso com áreas extensas de consolidação alveolar.

\section{RELATO DO CASO}

Homem de 16 anos, procedente de Medina, MG, estudante, não tabagista, previamente hígido. Foi internado, com relato de ter iniciado, havia dez dias, quadro de tosse seca, febre alta $\left(39^{\circ} \mathrm{C}\right)$, mal-estar, mialgia, dor abdominal difusa, queda do estado geral, dor em região escapular esquerda que evoluiu para dor torácica ventilatório-dependente e taquipnéia progressiva. À admissão, encontrava-se febril $\left(38,7^{\circ} \mathrm{C}\right)$, taquicárdico $(\mathrm{FC}=120 \mathrm{bpm})$, taquipnéico ( $\mathrm{FR}=28 \mathrm{ipm})$, ausculta pulmonar revelando sons respiratórios reduzidos e crepitações em ambas as bases, hepatomegalia dolorosa (fígado a $8 \mathrm{~cm}$ do rebordo costal direito), esplenomegalia Boyd I. A radiografia de tórax mostrava infiltrado intersticial difuso. A gasometria arterial revelava hipoxemia com baixa saturação de oxigênio $\left(\mathrm{pH}=7,37, \mathrm{PaO}_{2}=51 \mathrm{mmHg}, \mathrm{PaCO}_{2}=36 \mathrm{mmHg}\right.$, SatO 2 $\left.=85 \%, \mathrm{HCO}_{3}=20 \mathrm{mmol} / \mathrm{l}, \mathrm{BE}=-4 \mathrm{mmol} / \mathrm{l}\right)$. O hemograma mostrava hemoglobina $=12,1 \mathrm{~g} / \mathrm{dl}$; hematócrito $=$ $34 \%$; leucócitos totais $=2.800 / \mathrm{mm}^{3}$; bastonetes $=10 \%$, segmentados $=67 \%$; eosinófilos $=1 \%$; basófilos $=0$; linfócitos $=19 \%$; monócitos $=3 \%$. Uréia $=38$, creatinina $=0,6$.

A hipótese de diagnóstico foi de pneumonia comunitária grave e, então, iniciados ceftriaxona e claritromicina. Doze horas após a admissão, evoluiu com piora importante do padrão respiratório, sendo transferido para o Centro de Terapia Intensiva (CTI), onde progrediu para

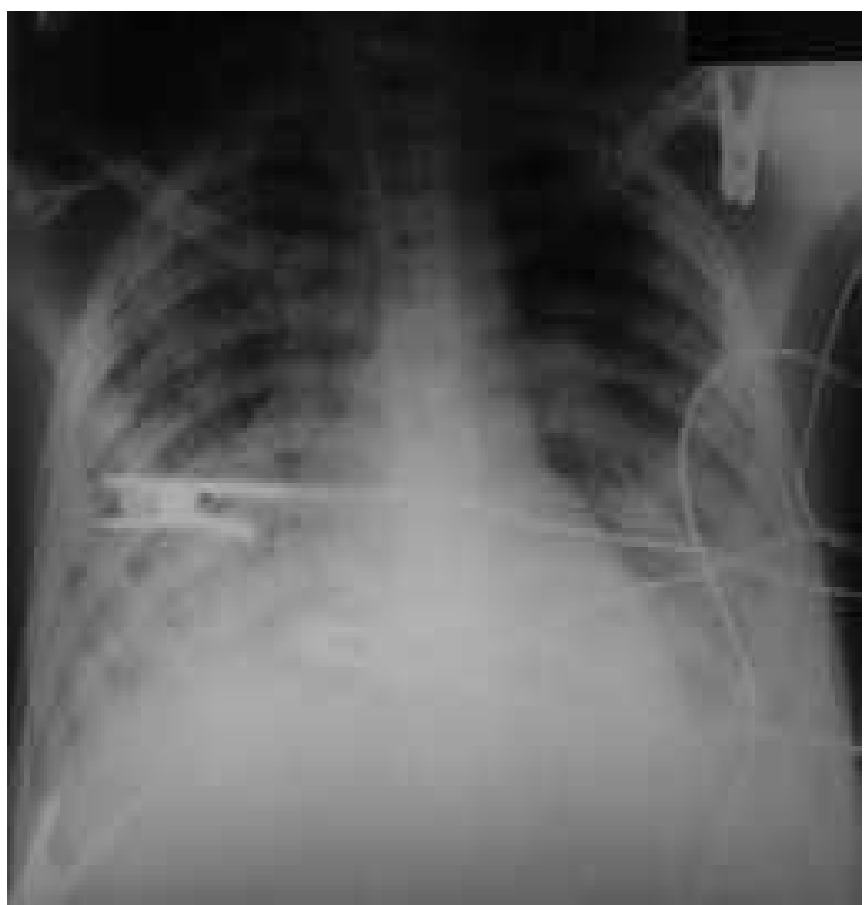

Figura 2 - Pneumonia por Chlamydia psittaci. Radiografia de tórax do paciente no CTI, em ventilação mecânica, com quadro de SARA (síndrome da angústia respiratória aguda) já estabelecido.

insuficiência respiratória aguda e síndrome da angústia respiratória do adulto (SARA), sendo intubado, colocado em ventilação mecânica. Foram acrescentados oxacilina, gentamicina e sulfametoxazol-trimetoprim. O paciente evoluiu com instabilidade hemodinâmica, piora do padrão radiológico, miose e ptose palpebral no olho esquerdo.

Os familiares relataram que o paciente possuía pequena criação de pombos. Foi adicionada ao esquema de antibióticos doxiciclina e suspenso sulfametoxasol-trimetoprim, colhida secreção traqueal para pesquisa de Chlamydia e sorologia para HIV. O paciente evoluiu com melhora do quadro e boa resposta antimicrobiana, sendo extubado oito dias após. Chlamydia psittaci foi detectada na secreção traqueal através da imunofluorescência com anticorpo monoclonal. A sorologia para HIV foi negativa. O paciente recebeu alta do CTI, sendo transferido para a enfermaria, onde apresentou boa evolução, porém, mantendo miose e ptose palpebral esquerda. Teve alta hospitalar 23 dias após a internação. Quarenta e cinco dias após a alta, o paciente apresentava bom estado geral, regressão das alterações oculares e padrão radiológico com melhora importante.

\section{DISCUSSÃO}

O agente causal da psitacose é a Chlamydia psittaci, germe gram-negativo, hospedeiro intracelular obrigatório $^{(6-9)}$. A transmissão para humanos dá-se através do con- 


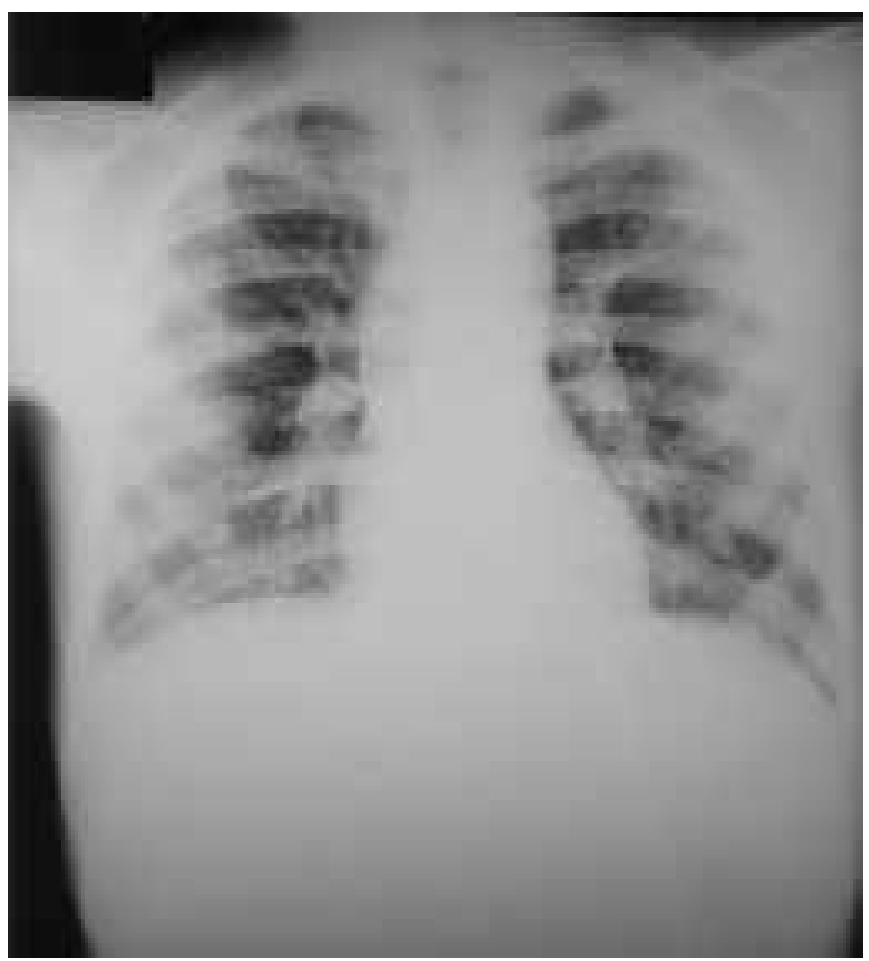

Figura 3 - Pneumonia por Chlamydia psittaci. Radiografia de tórax do paciente em alta hospitalar, mostrando melhora significativa do quadro pulmonar.

tato com pássaros, o qual é relatado em até $85 \%$ dos casos. Essa é a informação de maior valor diagnóstico à admissão(4,5). Exposições breves com aves também podem causar infecção sintomática e, em $15 \%$ dos casos, não se registra qualquer tipo de contato com esses animais. A Chlamydia psittaci infecta pássaros e animais domésticos, sendo excretada em grande número nas fezes, urina, secreções, saliva e penas ${ }^{(8,10,11)}$. A doença em humanos é adquirida pela inalação do microrganismo presente nas secreções ou fezes secas dos pássaros infectados $^{(2-4,8)}$. Outras maneiras de transmissão incluem bicadas, contato boca-bico, manuseio de penas e tecidos infectados. Pássaros recém-adquiridos representam importante modo de infecção, pois a doença nesses animais pode ser induzida por situações de estresse, como fome, superlotação, transporte e maus tratos. Se não tratadas, $10 \%$ das aves infectadas tornam-se portadoras assintomáticas. Transmissões inter-humanos são extremamente raras e tendem a ser mais graves ${ }^{(9)}$. A Chlamydia psittaci pode também infectar ovelhas, bodes e gado, causando infecção crônica no aparelho reprodutivo, insuficiência placentária e aborto nesses animais, mas a contaminação humana pela ingestão da carne infectada é rara ${ }^{(2,5)}$.

A doença começa a manifestar-se após período de incubação de 5-15 dias. O início geralmente é insidioso, com sintomas brandos, inespecíficos, lembrando infecção de vias aéreas superiores. Febre, tosse seca, cefaléia, calafrios, mialgia e hepatesplenomegalia ocorrem em mais da metade dos casos. Pode ocorrer o acometimento de vários órgãos, dentre eles o pulmão, trato gastrintestinal e sistema nervoso.

O órgão humano mais acometido é o pulmão, manifestando-se como tosse seca e dispnéia ${ }^{(12)}$. A manifestação típica é de pneumonia seguida a exposição às aves. Dor pleurítica é rara. Pneumonia confirmada radiologicamente é encontrada em até $80 \%$ dos casos. Não existem características radiológicas que permitam diferenciar a pneumonia por psitacose da pneumonia por outras causas. Os achados mais freqüentes são consolidação segmentar (31\%) e consolidação lobar (21\%), as quais ocorrem principalmente nos lobos inferiores. Derrame pleural é raro e, quando presente, é assintomático e pequeno ${ }^{(13)}$. Anormalidades neurológicas são sintomas raros da doença. Já foram descritos distúrbios cerebelares, mielite transversa, meningite, confusão mental, hipertensão intracraniana, sindrome de Guillain-Barre, paralisia de pares cranianos, entre eles II, III, IV, VI, VII e XII ${ }^{(14-19)}$. As manifestações gastrintestinais mais freqüentes são vômitos, diarréia, anorexia e dor abdominal. A psitacose é raramente doença sistêmica grave e fulminante $e^{(3,12)}$.

Não existem achados laboratoriais específicos. A contagem de leucócitos geralmente é menor que $10.000 \mathrm{em}$ $2 / 3$ dos pacientes. Podem ocorrer anormalidades na função hepática. Hipoxemia e hipercapnia são achados nos casos graves e estão associados a pior prognóstico(3). Cultura do organismo é possivel, porém pouco viável, devido à natureza esporádica da doença, ao risco de contaminação e à dificuldade em obter material adequado para a cultura ${ }^{(10,15)}$. O diagnóstico sorológico pode ser confirmado em até $94 \%$ dos casos, mas são descritas, na literatura, reações cruzadas. Teste de fixação com complemento é usado, mas é somente gênero-específico $e$ não distingue C. psittaci de C. trachomatis ou C. pneumoniae $^{(20)}$. Técnicas mais recentes, como reação em cadeia de polimerase (PCR) e detecção de antígeno através de imunofluorescência com anticorpo monoclonal, são as preferidas por ser métodos rápidos, confiáveis e de grande especificidade (20-24). $^{2}$

Os principais diagnósticos diferenciais da psitacose são outras causas de pneumonia atípica. Mycoplasma pneumoniae, Chlamydia pneumoniae e Legionella pneumophila são as causas mais comuns ${ }^{(25,26)}$. As tetraciclinas, especialmente a doxiciclina, constituem o tratamento de escolha da psitacose. A terapêutica precoce evita casos graves. Após o início da droga, os sintomas tendem a regredir em 24 a 48 horas, embora a melhora possa ser lenta em alguns casos. Recomenda-se tratamento por 21 dias, para evitar recorrências. A eritromicina é uma for- 
ma alternativa de tratamento, porém apresenta baixa resposta quando a infecção é grave $e^{(3,4,6)}$.

Os pássaros infectados devem ser tratados com tetraciclina ou doxiciclina pelo menos por 45 dias. Nos países desenvolvidos, as aves importadas recebem tetraciclina em regime de quarentena e depois usa-se adicionalmente por mais 15 dias, antes de ser comercializadas. Alguns autores recomendam profilaxia com doxiciclina durante dez dias, para contatos de pacientes infectados ${ }^{(19,27)}$.

A psitacose é diagnóstico diferencial importante a ser considerado em situações de pneumonia comunitária que não responde à antibioticoterapia convencional, que evolui de modo insatisfatório e cuja epidemiologia é positiva para exposição às aves. O tratamento precoce é fundamental no prognóstico devido à boa resposta terapêutica ${ }^{(24,27)}$.

\section{AgRAdECIMENTOS}

Os autores agradecem ao Dr. José Carlos Serufo e ao laboratório MICRA, pela contribuição na investigação diagnóstica deste caso.

\section{REFERÊNCIAS}

1. Vanropay D, Ducatelle R, Haesebrouck F. Chlamydia psittaci infections: a review with emphasis on avian chlamydiosis. Vet Microbiol 1995; 45:93-119.

2. Butler JC, Whitney CG. Compendium of measures to control Chlamydia psittaci infection among humans (psittacosis) and pet birds (avian chlamydiosis). MMWR Morb Mortal Wkly Rep 1998;47:1-14.

3. MacFarlane JT, Markey B, Gleeson M, et al. Psittacosis. Med Bull 1983; 39:163-167.

4. Yung AP, Grayson ML. Psittacosis: a review of 135 cases. Med J Aust $1988 ; 148: 228-233$.

5. Centers for Disease Control and Prevention. Compendium of Psittacosis (chlamydiosis) Control, 1997. MMWR Morb Mortal Wkly Rep 1997; 46(RR13):1-13.

6. Verweij PE, Meis JF, Eijk R, Melchers WJ. Severe human psittacosis requiring artificial ventilation: case report and review. Clin Infect Dis 1995;20:440-442.

7. Herring AJ. The molecular biology of chlamydia - A brief overview. J Infect 1992;25:1-10.

8. van Buuren CE, Dorrestein GM, van Dijk JE. Chlamydia psittaci infections in birds: a review on the pathogenesis and histopathological features. Vet Q 1994;16:38-41.
9. Peeling RW, Brunham RC. Chlamydiae as pathogens: new species and new issues. Emerg Infect Dis 1996;4:307-319.

10. Centers for Disease Control. Psittacosis surveillance. Annual summary. Atlanta: Centers for Disease Control, 1987.

11. Reeve RVA, Carter LA, Taylor N, et al. Respiratory tract infections and importation of exotic birds. Lancet 1998;829-830.

12. Riantawan P, Nunthapisud P. Psittacosis pneumonia: a case and review of the literature. J Med Assoc Thai 1996;79:55-59.

13. Shee CD. Cerebellar disturbance in psittacosis. Postgrad Med 1988; 64:381-383.

14. Sunderland R, Williams W. As sick as a pigeon - Psittacosis myelitis. Arch Dis Child 1989;64:1626-1628.

15. Crook T, Bannister B. Acute transverse myelitis associated with Chlamydia psittaci infection. Infect 1996;32:151-152.

16. Newton P, Lalvani A, Conlon CP. Psittacosis associated with bilateral $4^{\text {th }}$ cranial nerve palsies. Infect $1996 ; 32: 63-65$.

17. Brewis C, Chir B, McFerran DJ. Farmers ear: sudden sensorial hearing loss due to Chlamydia psittaci infection. N Laryngol Otol 1997; 111:855-857.

18. Zumla A, Lipscomb G, Lewis D. Sixth cranial nerve palsy complicating psittacosis. J Neurol Neurosurg Psychiatry 1988;51:1462.

19. Coutts II, Mackenzie S, White RJ. Clinical and radiographic features of psittacosis infection. Thorax 1985;40:530-532.

20. Sillis M, White PMB. Rapid identification of Chlamydia psittaci and TWAR (C. pneumoniae) in sputum samples using an amplified EIA. J Clin Pathol 1990;43:260.

21. Fukushi H, Nojiri K, Hirai K. Monoclonal antibody typing of Chlamydia psittaci strains derived from avian and mammalian species. Clin Microbiol 1987;25:1978-1981.

22. Messmer TO, Skelton SK, Moroney JF, et al. Application of a nested, multiplex PCR to psittacosis outbreaks. Clin Microbiol 1997;35:20432046.

23. Kauppinen M, Sakku P. Pneumonia due to Chlamydia pneumoniae: prevalence, clinical features, diagnosis and treatment. Clinical Infect Dis 1995;21:244-252.

24. File TM, Tan JS, Plouffe JF. The role of atypical patogens: Mycoplasma pneumoniae, Chlamydia pneumoniae, and Legionella pneumophila in respiratory infection. Infect Dis Clin North Am 1998;12:569591.

25. Cunha AB, Ortega AM. Atypical pneumonia - Extrapulmonary clue guide the way to diagnosis. Postgrad Med 1996;99:123-132.

26. Oldach DW, Gaydos CA, Mundy LM, Quinn TC. Rapid diagnosis of Chlamydia psittaci pneumonia. Clin Infect Dis 1993;17:338-343.

27. Byrom NP, Walls J. Fulminant psittacosis. Lancet 1979;i:353-356. 